\title{
BASAMENTO HISTÓRICO-METODOLÓGICO DE SISTEMATIZACIÓN DE LA OBRA PEDAGÓGICA DE PERSONALIDADES RELEVANTES DEL SIGLO XX Y/O XXI: UNA PERSPECTIVA DESDE LA HERMENÉUTICA
}

\author{
PhD. Lidia Turner Martí \\ Universidad de Oriente \\ lidia@rimed.cu
}

\author{
PhD. Idalberto Senú González \\ Universidad de Oriente \\ idalberto.senu@uo.edu.cu
}

\author{
PhD. Juana Cubela González \\ Universidad de Oriente \\ jmcubela@uo.edu.cu
}

Palabras claves: basamento histórico-metodológico, comprensión,

Recibido: 10 de febrero de 2017

interpretación.

Keywords: methodological-Historical basement, comprehension, explanation.

Aceptado: 17 de Marzo de 2017

\section{RESUMEN}

En este artículo se presenta una construcción teórica consistente en un basamento histórico-metodológico para el estudio de la obra pedagógica de destacadas personalidades de la educación, que permite enriquecer la Historia de la Pedagogía de cualquier país. Se centra en una categoría esencial: las unidades de análisis de contenido hermenéutico, dicho constructo permite el análisis comprensivo, explicativo e interpretativo de la actuación profesional pedagógica de la personalidad que se desea estudiar. El redimensionamiento de estos procesos coadyuvó a los autores a proponer, desde un contenido hermenéutico, el basamento teórico-metodológico que sirve de sostén para poder llegar a la sistematización de la obra pedagógica de relevantes educadores del siglo $X X$ y XXI, revelando las cualidades que la distinguen y su trascendencia en el proceso educativo o en la formación del alumno.

\section{ABSTRACT}

In this article it is introduced a theoretical construction the enrich the History of Pedagogy, denominated as historical-methodological basement, starts of focusing on the essential category the unit of analysis of the hermeneutic content, having a bases this construct that allows to determines, to study and analysis other personalities. There arrangement of these processes lead this author to propose he unities of analysis of the hermeneutic content as theoretical foundation he serves as support to reach systematization of relevant educator's pedagogical work of the XX century, it rebels the qualities that distinguish and its transcendence in educator's training. 


\section{INTRODUCCIÓN}

La Historia de la Pedagogía cuenta con la obra de destacados educadores, cuyas reflexiones y experiencias pedagógicas de avanzada permiten dar continuidad a las mejores tradiciones del magisterio para enriquecer la formación de las presentes y futuras generaciones. Muchos investigadores han impulsado las investigaciones de corte histórico-pedagógico, entre los que se destacan Chávez (1990-1991-19921994-1996-2003); Ramos (2003); Buenavilla (1995-1997-2002-2004); Senú (2016), entre otros.

Las raíces históricas que han condicionado las prácticas pedagógicas en todos los tiempos, permite estudiar los antecedentes históricos de la educación. Reconstruir la memoria del magisterio es una tarea esencial en la actualidad, ella es la síntesis de todo el quehacer pedagógico a través del tiempo.

Proponer un basamento histórico-metodológico de sistematización de la obra pedagógica de personalidades relevantes del siglo $X X$ y/o XXI, desde la hermenéutica, constituye el objetivo esencial de este artículo, lo cual resulta consustancial para la sistematización, enriquecimiento y reconocimiento del pensamiento y la práctica educativa de aquellos educadores que con su labor pedagógica perpetuán la formación de las futuras generaciones.

\section{DESARROLLO}

En la actualidad es recurrente escuchar el término hermenéutica, proviene del verbo griego hermenéuticos que significa interpretar, declarar, anunciar, esclarecer y traducir. Significa que alguna cosa es vuelta comprensible o llevada a la comprensión, los estudios hermenéuticos han evolucionado por diferentes períodos y momentos históricos, hasta la actualidad.

Se sostiene la idea de que la hermenéutica, se erige en el arte o la ciencia de la interpretación, por lo que es importante tener en consideración los tres procesos interpretativos del significado hermenéutico, según las conceptualizaciones abordadas por Fuentes; Matos y Montoya (2007) y otros, redimensionadas por los autores para la interpretación y estudio de los aportes de una determinada personalidad pedagógica.

Estos procesos son:

- Comprensión: es el modo de aprehensión de los sujetos de la cultura, es un método para un primer acercamiento al significado, y constituye una imagen totalizadora del objeto de investigación. Se sustenta en lo fáctico, en el análisis histórico-tendencial y en la caracterización epistemológica de dicho objeto.

- Explicación: en la explicación se significan hechos, manifestaciones y las relaciones causales (dialécticas) en el comportamiento del objeto de investigación. Es un segundo nivel de interpretación de la hermenéutica, y la forma de encontrar razones de la significación del objeto, constituye un análisis, que es más esencial que la comprensión al fragmentar las causas, posibilita penetrar con mayor profundidad en la esencia del comportamiento de investigación.

- Interpretación: constituye el desarrollo y realización de la comprensión y la explicación, como síntesis de ellas y permite la reconstrucción del 
significado del objeto de investigación, adquiriendo con ello un sentido diferente y cualitativamente superior". (Fuentes, Matos, y Rivera., 2007, p. 139-140). Estos investigadores defienden el empleo del enfoque metodológico hermenéutico dialéctico, lo cual es asumido por los autores al abordar la sistematización de una obra pedagógica.

Dichos autores ofrecen una importante reflexión: "La hermenéutica es aún poco conocida, requiere de estudios profundos, científicos y a menudo se encuentra conceptualizada muy restringida pero negar su valor en las investigaciones científicas es privar al investigador de un enfoque, una teoría y un método que ofrece un instrumental para descifrar, desde la subjetividad humana, un objeto de investigación, interpretarlo y a su vez, transformarlo". (Fuentes, Matos, y Rivera, 2007,p.141).

En la investigación hermenéutica ha de ser analizada la situación histórico-socialcultural en que se produce la obra pedagógica de la personalidad, y por tanto, sus construcciones semánticas. Ello propicia un proceso activo de búsqueda, constatación, análisis, comprensión e interpretación a través del cual se va desarrollando una descripción progresiva de los hechos, sucesos, acontecimientos, incidencias y aportaciones que trascienden en su próspera vida pedagógica.

La búsqueda hermenéutica debe caracterizarse por su carácter cualitativo, flexible, abierto, interactivo y constructivo donde se retomaron una y otra vez sus escritos, entrevistas, testimonios, apuntes, investigaciones para llegar a una interpretación profunda y objetiva de su trascendencia en el proceso la formativo o en el desarrollo del fenómeno educativo integralmente, lo que conduce a comprender el conocimiento de su obra desde los significados y los sentidos que tiene para la personalidad investigada y los sujetos con los cuales se relacionó y formó.

Las razones expuestas permiten visualizar por qué se hizo imprescindible retomar la hermenéutica como esencia y fundamento en el estudio de personalidades, redimensionando, desde una interrelación dialéctica, los procesos de comprensión, explicación e interpretación para poder llegar a una sistematización de la obra estudiada y su trascendencia pedagógica.

La comprensión favorece tener un primer acercamiento al significado de la obra, una imagen totalizadora de su práctica educativa y su pensamiento pedagógico desde un nivel fáctico, el análisis histórico-tendencial y la caracterización de su personalidad y quehacer pedagógico.

La explicación debe ser utilizada con la intención de significar los hechos, manifestaciones y las relaciones causales en el comportamiento de toda la obra pedagoga de la personalidad y en la construcción de sus aportaciones a la Pedagogía, al proceso educativo o formativo multidimensionalmente.

Es en un segundo nivel de interpretación hermenéutica, donde se encuentran las razones de la significación de su obra pedagógica. El análisis causal y la explicación de las razones posibilitan penetrar con mayor profundidad en la esencia de la investigación.

La interpretación permite el desarrollo y la realización de la comprensión y la explicación de su obra pedagógica, para poder hacer la sistematización de esta, como síntesis de sus aportaciones a la Pedagogía, a la vez que permite la reconstrucción de su significado adquiriendo con ello un alcance cualitativamente superior. 
El redimensionamiento de estos procesos de comprensión, explicación e interpretación para poder realizar el estudio de sistematización de la obra pedagógica de la personalidad, coadyuvó a proponer las unidades de análisis del contenido hermenéutico como constructo teórico-metodológico que sirve de sostén para poder llegar a la sistematización de la obra, revelar las cualidades que la distinguen y su trascendencia en la educación.

Se parte de considerar las unidades de análisis como los elementos en los que recae la obtención de la información requerida, lo que supone varios procesos simultáneos de recolección, análisis y procesamiento, y por consiguiente, de una mayor exigencia analítica para establecer relaciones, determinar causales, establecer razones, todo lo cual es imprescindible para el tema abordado. En este sentido, las unidades de análisis bajo los presupuestos del paradigma hermenéutico, posibilitan hacer una interpretación de la vida y obra pedagógica de la personalidad, con mayores niveles de análisis, comprensión y sistematización.

Lo expresado anteriormente permitió definir como una categoría esencial: las unidades de análisis del contenido hermenéutico como eje dinamizador del proceso de sistematización: Se asume el análisis hermenéutico desde el paradigma interpretativo comprensivo; lo que supone un rescate de los elementos que distinguen la obra construida por la personalidad que se investiga, sobre aquellos hechos externos a ella. En este sentido, se destaca como eje fundamental el proceso de interpretación. El análisis hermenéutico como expresión del proceso investigativo se sustenta en un estudio totalizador de su obra e intenta visualizar su pensamiento y práctica pedagógica dentro de un horizonte general y una interpretación profunda de su quehacer pedagógico, a partir de un método que asuma a su vez dos perspectivas de desarrollo:

- $\quad$ Una interpretación totalizadora de su vida y obra que abarque el contenido preliminar de estas desde su observación, comprensión, explicación e interpretación de sus prácticas, ideas, concepciones y enfoques pedagógicos relacionados con los educadores.

- $\quad$ Otra relacionada con sus componentes o configuraciones que revelen sus principales rasgos, cualidades y relaciones esenciales, desde una perspectiva globalizadora del objeto.

Este proceso se inicia con la representación de un todo no fragmentado que da tránsito al análisis de sus partes o expresiones y finaliza con la reconstrucción de ese objeto, ya transformado desde una representación totalizadora, la cual se caracteriza por nuevas cualidades que no son resultado de las partes en sí mismas, sino síntesis de su interrelación dentro del sistema de conexiones que se dan entre ellas.

Desde esta perspectiva se propone el basamento histórico-metodológico: las unidades de análisis del contenido hermenéutico para la sistematización de la obra pedagógica de la personalidad que se desea estudiar. Se basa en el análisis comprensivo, aclarativo, explicativo e interpretativo de su actuación profesional pedagógica, de las construcciones teóricas que la distinguen y su trascendencia en otros profesionales por su alcance y efecto transformador en la práctica educativa.

De manera que, se centra como categoría esencial: las unidades de análisis del contenido hermenéutico, las que se erigen en constructos de análisis históricopedagógico en relación con el análisis lógico teórico, mediado por el proceder interpretativo, a partir de la comprensión de la vida pedagógica y la explicación de 
la obra pedagógica de la personalidad investigada, esos son los elementos, que interconectados dialécticamente, viabilizan el análisis del contenido interpretativo, lo cual da cuenta de una visión nueva en la indagación lógica del pensamiento pedagógico y la revelación histórica de la práctica educativa de la personalidad, quien desarrolla su vida en períodos históricos y contextos que marcan la impronta de su quehacer pedagógico, constituyendo un ejemplo para futuras generaciones.

Este constructo permite estudiar las diferentes personalidades que se destacan por su hacer o desempeño pedagógico, entonces se constituye en un aporte a la teoría y la historiografía en las ciencias pedagógicas, que hasta la actualidad no ha sido revelado desde el criterio de este constructo esencial.

Para una mayor comprensión de la lógica sistémica asumida se enfatiza en que el análisis histórico no se reduce a una simple descripción empírica de los hechos acontecidos, sino que recoge los aspectos necesarios de la esencia de la personalidad estudiada en relación con las formas histórico-concretas en que desarrolla su vida.

En este sentido, el constructo análisis histórico pedagógico, permite el estudio del fenómeno pedagógico en su desarrollo histórico, donde va adoptando diversas peculiaridades y formas de manifestación. Se reconoce, desde esta propuesta, que el análisis profundo de las características y tendencias de la época o momento histórico en que se desenvuelve la actuación de la personalidad resulta necesario para significar sus aportes o contribuciones al desarrollo de la sociedad, la educación, al pensamiento pedagógico.

Por su parte, el análisis lógico teórico "supera" el análisis histórico de sus aspectos causales. Se orienta a considerar lo lógico y lo teórico. El análisis histórico es punto de partida y fundamento del análisis lógico, pero lo lógico no se corresponde con lo histórico cuando el razonamiento solo opera a un nivel empírico, sino cuando se acompaña del análisis teórico que consiste en un análisis sistémico e histórico concreto de la esencia humana de la personalidad que se forma y evoluciona, expresándose la unidad de lo histórico y lo lógico.

En correspondencia entre lo histórico-lógico y el lógico-teórico, como parte de este estudio, se refiere exclusivamente a las leyes del proceso real. Todo ello mediado por el proceder interpretativo de la personalidad.

En tal sentido, el proceder interpretativo de la personalidad es entendido como el camino que se ha de seguir para el estudio de personalidades representativas, a partir de un análisis profundo y objetivo de lo histórico y lógico, proceso contentivo de un sistema de acciones histórico, lógico-metodológicas y prácticas que se encaminan a la interpretación hermenéutica del sentido y significado de las personalidades o educadores y su trascendencia en la educación u otros procesos pedagógicos que tienen que ver con la formación del educando.

En la relación sistémica del proceder interpretativo emergen como aspectos estructurales: la comprensión de la vida pedagógica; la explicación de obra formativa del sujeto y el análisis del contenido interpretativo de la vida y obra como componente de síntesis en este proceso.

La comprensión de la vida pedagógica es el proceso que permite una indagación inicial para obtener una familiarización con los factores de carácter histórico, social, económico, político y educacional que alcanzan significado en la historia de vida de 
la personalidad, garantizando el razonamiento y conocimiento sobre los momentos más trascendentales vividos por esta y por qué repercuten en su formación profesional y en la conducción de instituciones educativas o procesos pedagógicos. Ello requiere del estudio de su biografía o autobiografía, testimonios, historia de vida, análisis documental, entre otros métodos de indagación personal y personalizada, dando pie a una cronología y periodización de su vida y obra pedagógica.

La comprensión de la vida pedagógica conduce a un primer acercamiento al significado de la obra, una imagen totalizadora de su práctica educativa y su pensamiento pedagógico desde un nivel fáctico, del análisis histórico-tendencial, la caracterización de su personalidad y quehacer pedagógico.

La explicación de la obra formativa de la personalidad permite complementar dialécticamente el conocimiento adquirido con la comprensión anterior, para llegar a revelar las relaciones causales en el comportamiento de toda su obra pedagógica y la dilucidación de sus aportes a la formación de profesionales.

El análisis y la explicación causal viabilizan la profundización en el estudio de la personalidad. Esos elementos explicados dan cuenta del análisis del contenido interpretativo, con una visión nueva, desde lo teórico, en la indagación lógica del pensamiento pedagógico y la revelación histórica de la práctica educativa, realzándose el estudio de su vida y obra formativa.

Las contribuciones teóricas y aportes presentes en obra de la personalidad, una vez sistematizados y divulgados no solo trascienden, sino que pueden ser generalizados e introducidos a la práctica social y educativa, impactando en las generaciones que formó y en las nuevas generaciones de profesionales que, inspirados en sus concepciones, enfoques e ideas revolucionarias, se motivan por hacerlas realidad en la formación armónica de niños, adolescentes y jóvenes.

\section{CONCLUSIONES}

La fundamentación del basamento histórico-metodológico sobre las unidades de análisis de contenido hermenéutico puede ser utilizado para la sistematización de la obra pedagógica de una personalidad relevante, desde esta nueva mirada metodológica, posibilita realizar el registro, ordenamiento, comprensión, explicación e interpretación de las claves de contenido que distinguen su pensamiento pedagógico y la práctica educativa relacionada con el proceso educativo o la formación del alumno.

Las relaciones dialécticas que emergen del basamento histórico-metodológico propuesto, adquieren su máxima expresión en las unidades de análisis del contenido hermenéutico como eje que dinamiza todo este proceso, lo cual demuestra un mayor nivel de esencialidad en la sistematización del estudio de la vida y obra de personalidades destacadas del siglo XX y/o XXI. 


\section{REFERENCIAS BIBLIOGRÁFICAS}

Buenavilla, R. (2004). Metodología de la investigación de la vida y obra pedagógica de destacados educadores. Proyecto de investigación contribución de destacadas figuras de la cultura nacional al desarrollo de la educación cubana. Instituto Superior Pedagógico Enrique José Varona. Facultad de Ciencias de la Educación. Enero -Diciembre.2004.

Chávez, A. (1996). Bosquejo histórico de las ideas educativas en Cuba. La Habana: Editorial Pueblo y Educación.

Fuentes, H.; Matos, E. y Montoya, J. (2007). El proceso de investigación científica orientada a las investigaciones en Ciencias Sociales. Universidad Estatal de Bolívar, Guaranda.

Ramos, G. (2003). La sistematización de las concepciones pedagógicas predominantes durante el período neocolonial en Santiago de Cuba. (19021958). Tesis en opción al grado científico de Doctor en Ciencias Pedagógicas. UCP, Instituto Superior Pedagógico Frank País García, Santiago de Cuba.

Senú, I.; Miranda, A. y Cubela, J. (2015). Estrategia pedagógica para promover el conocimiento de la vida y obra pedagógica de la maestra Lidia Esther Turner Martí en la asignatura Historia de la Pedagogía. Trabajo Final Especialidad de Postgrado en Docencia Psicopedagógica. Universidad de Ciencias Pedagógicas Frank País García, Facultad de Ciencias de la Educación, Departamento de Pedagogía- Psicología, Santiago de Cuba.

Senú, I. (2016). Contribución de la obra pedagógica de Lidia Esther Turner Martí a la formación de los profesionales de la educación. Tesis presentada en opción al grado científico de Doctor en Ciencias Pedagógicas. Universidad de Oriente, Facultad de Educación Infantil, Departamento de PedagogíaPsicología, Santiago de Cuba. 